\title{
Topology of two-band superconductors
}

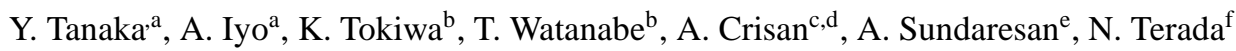 \\ ${ }^{a}$ National Institute of Advanced Industrial Science and Technology, Tsukuba, Japan \\ ${ }^{b}$ Tokyo University of Science, Noda, Japan \\ ${ }^{c}$ National Institute of Materials Physics, Bucharest, Romania \\ ${ }^{d}$ University of Birmingham, Birmingham, $U K$ \\ e Jawaharlal Nehru Centre for Advanced Scientific Research, Bangalore, India \\ ${ }^{f}$ Kagoshima University, Kagoshima, Japan
}

\begin{abstract}
Two-band superconductivity has a topology different from that in single-band superconductivity. The topology is not always stabilized in an infinitely homogeneous sample. The morphology, grain shape, and pattern of the device (topology of the superconducting materials) is effective in stabilizing the topology. In this report, we discuss a vortex having a small magnetic flux but a large winding number as one plausible topology in a two-band superconductor.
\end{abstract}

Key words: Two-band superconductor, Multilayer cuprate superconductor, interband phase difference soliton PACS: 74.90.+n, 74.40.+k, 74.20.De, 74.25.Qt

\section{Introduction}

Since the 1950s, many two-band superconductors (and multi-band superconductors) have been investigated experimentally and theoretically. These include transition metals such as $\mathrm{Nb}$, graphite intercalation compounds such as $\mathrm{KC}_{8}$, intermetallic rare earth transition metal borocarbides such as $\mathrm{YNi}_{2} \mathrm{~B}_{2} \mathrm{C}$, multilayer cuprate superconductors such as $\mathrm{Cu}_{x} \mathrm{Ba}_{2} \mathrm{Ca}_{3} \mathrm{Cu}_{4} \mathrm{O}_{y}$, magnesium diboride, iron-based superconductors such as $\mathrm{NdFeAsO}_{1-x}$, and so on $[1,2]$.

In two-band superconductors, we can consider that each superconducting condensation on each band has its own superconducting phase. This consideration can be applied when the interband interaction is much smaller than the intraband interaction. Using an extended London approximation, in which we assume that the superconducting pair density can be considered constant and no spatial gradient exists, the properties of the system are governed by the interband phase difference. This situation gives an intuitive image of the physics involved [3, 4].

\section{Model}

We substitute two-component order parameter, $\left\{\left|\Psi_{1}\right| \exp \left(i \theta_{1}\right),\left|\Psi_{2}\right| \exp \left(i \theta_{2}\right)\right\}$ for the usual order parameter, $\Psi$, used in single-band superconductivity $[1,2,3,4,5,6,7,8,9]$. $\left|\Psi_{v}\right|^{2}$ is the pair density on each band index by $v$, which is assumed to be constant (an extended London approximation). $\theta_{v}$ is the quantum phase of each component.

When there is no magnetic field, the current $J=\frac{e n_{1} \hbar}{m_{1}} \nabla \theta_{1}+$ $\frac{e n_{2} \hbar}{m_{2}} \nabla \theta_{2}$ becomes zero (this current can be called the noninductive current) $[2,4,5]$. This condition gives the relationship

Email address: y.tanaka@aist.go.jp (Y. Tanaka) between the two phases for two bands. When there is a real current inducing a magnetic field (an inductive current), the phase change owing to this real current is the same in both bands. The total phase is the sum of the phases due to the non-inductive and inductive currents $[2,4,5]$.

\section{Result}

At the ground states, $\theta_{1}=\theta_{2}$. When there is an interband phase difference soliton, this soliton (or more precisely, the soliton wall) divides the superconductor into two regions having different quantum phases (Formation of the soliton is irrelevant to the magnetic field.) $[1,4,6]$. The trace of the phase change is shown in Figs.1 (a) and (b) under zero magnetic field. Here, we trace one location to another on a continuously connected path in the two-band superconductor. $\theta_{1}=\theta_{2}$ corresponds to the phase before and after crossing the soliton. Other regions correspond to inside the soliton. On crossing the soliton, the phase changes $\Delta \theta_{1}= \pm 2 \pi \frac{\frac{m_{1}}{N_{1}}}{\frac{m_{1}}{N_{1}}+\frac{m_{2}}{N_{2}}}$ and $\Delta \theta_{2}=\mp 2 \pi \frac{\frac{m_{2}}{N_{2}}}{\frac{m_{1}}{N_{1}}+\frac{m_{2}}{N_{2}}}$.

\section{Discussion}

When $\left|\Psi_{1}\right|=\left|\Psi_{2}\right|$, the phase changes by $\pi$ when crossing a soliton. This situation is the same as in triplet superconductivity (pseudo-triplet superconductivity) [7, 8]. When we cross another soliton, the phase recovers $2 \pi$. Regions where $\theta_{\text {domain }}=\theta_{1}=\theta_{2}$ (the phase of a domain surrounded by the soliton wall) are classified into either $\theta_{\text {domain }}=0$ or $\theta_{\text {domain }}=\pi$.

When $\left|\Psi_{1}\right| \neq\left|\Psi_{2}\right|$ we cannot recover the phase even after crossing two soliton walls, unlike in a pseudo-triplet superconductivity. $\theta_{\text {domain }}$ can take other values than 0 and $\pi$ [7]. In 


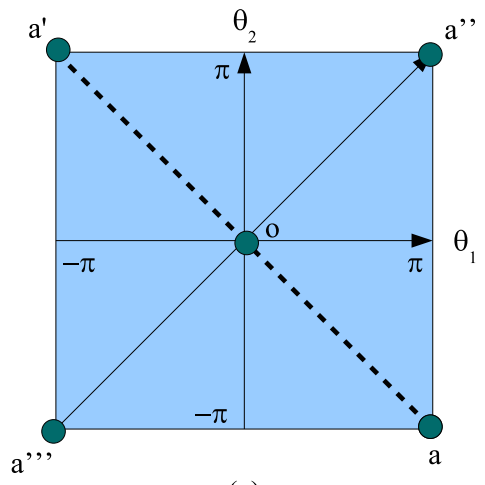

(a)

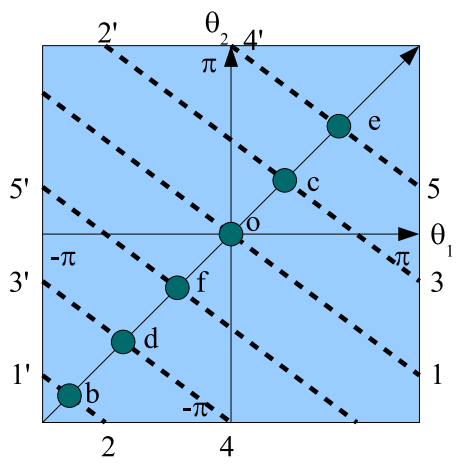

(b)

Figure 1: The trace of the phase. (a) Pseudo-triplet superconductivity. (b) General two-band superconductivity. When we cross a soliton wall, the phase of the path changes on the broken lines as $o \rightarrow 1 \rightarrow 1^{\prime} \rightarrow b \rightarrow 2$ and so on. 1 and 1' indicate the same point in phase space. The ground states are on the line $\theta_{1}=\theta_{2}$ (the phase changes on this line when a magnetic field is applied). a, a', $\mathrm{a}$ ", and a"' indicate the same points. $\theta_{1}=\theta_{2}$ at $\mathrm{o}, \mathrm{a}, \mathrm{b}, \mathrm{c}, \ldots$, and $\mathrm{f}$.

particular, when $\frac{\left|\Psi_{1}\right|}{\left|\Psi_{2}\right|}$ is an irrational number, we can never recover the original phase by crossing a finite number of solitons. We have to introduce an anti-soliton if we wish to revover the original phase.

This difference between pseudo-triplet superconductivity and the case where $\frac{\left|\Psi_{1}\right|}{\left|\Psi_{2}\right|}$ is an irrational number results in a difference in the flux quantization of a fractional vortex. The fractional vortex is the terminator of the soliton wall $[2,9]$. It introduces the magnetic flux and the vector potential accompanied by the circulating (inductive) currnet. The sum of the phase slip owing to the soliton and the phase change owing to the fractional flux quanta becomes $2 n \pi$ ( $n$ is an integer) [2, 4, 9].

One fractional vortex can terminate several soliton walls simultaneously, as shown in Fig.2. In pseudo-triplet superconductivity, the fractional flux quanta of $\frac{\Phi_{0}}{2}$ is sufficient to terminate any number of soliton walls (Here $\Phi_{0}$ is the usual flux quantum.) [7]. However, we need arbitrary fractional flux quanta to terminate an arbitrary number of soliton walls in the case where $\frac{\left|\Psi_{1}\right|}{\left|\Psi_{2}\right|}$ is an irrational number, as seen in Fig.1 (b).

This means that arbitrarily small flux quantization can be made possible by gathering the many number of soliton walls. Moreover, the value of the flux quanta neither increases nor decreases monotonously with an increasing number of solitons.
The soliton wall can be pinned or trapped at thinner locations of the sample. We can also make such a trapping center artificially using nanotechnology. This trapped soliton walls breaks the flux quantization condition, and finally we may hardly see the normal full vortex. The soliton walls may mash a flux quanta.

When the other sides of each soliton wall are terminated by the sample edge, the fractional vortex becomes a joint center of the soliton walls, and the soliton walls become anchors. Though it has finite formation energy, a topological constraint may stabilize this vortex.

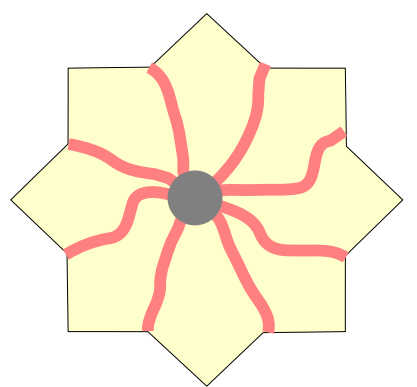

Figure 2: Monster vortex. The large point indicates the center of the fractional vortex. Bold lines indicate soliton walls pinned by dents in a grain.

\section{Summary}

In two-band superconductors, an abnormal vortex can be stabilized by the sample's morphology. This monster vortex can join many soliton walls and have arbitrarily small flux quanta.

\section{Acknowledgments}

This work was supported by the Strategic International Cooperative Program of the Japan Science and Technology Agency (JST) and the Department of Science and Technology (India) 'Feasibility study of the application of multiple-order parameters in materials to information processing'; by a Grantin-Aid for Specially Promoted Research (20001004) from The Ministry of Education, Culture, Sports, Science and Technology (MEXT); by the National Institute of Advanced Industrial Science and Technology (AIST) through a grant for overriding priority research 'New Superconducting Revolution'; by the Romanian Ministry of Education and Research; by the European Commission through the Marie Curie Excellence Grant 'NanoTechPinningHTS'.

\section{References}

[1] A. J. Leggett, Prog. Theor. Phys. 36 (1966) 901.

[2] Y. Tanaka et. al, Jpn. J. Appl. Phys. 46 (2007) 134, and references therein.

[3] Y. Tanaka, J. Phys. Soc. Jpn. 70 (2001) 2844.

[4] Y. Tanaka, Phys. Rev. Lett. 88 (2002) 017002.

[5] Y. Tanaka et. al, J. Phys. Conf. Ser. 150 (2009) 052267.

[6] A. Gurevich and V. M. Vinokur Phys. Rev. Lett. 90 (2003) 047004.

[7] Y. A. Izyumov and V. M. Laptev Phase Transit. 20 (1990) 95.

[8] Y. M. Cho and P. M. Zhang, Eur. Phys. J. B 65 (2008) 155.

[9] E. Babaev, Phys. Rev. Lett. 89 (2002) 067001. 